\title{
ROSAT Observations of Radio Quasars: Unification of Flat and Steep-Spectrum Sources?
}

\author{
W. Yuan, W. Brinkmann, and J. Siebert \\ Max-Planck-Institut für Extraterrestrische Physik, D-85740, Germany
}

\begin{abstract}
We have studied the dependence of X-ray spectral and luminosity properties on radio morphology using ROSAT data for a sample of radio quasars. The results support the unified schemes for FS and SS quasars.
\end{abstract}

\section{Introduction}

$\mathrm{X}$-ray observations have shown that flat-spectrum (FS) radio quasars have on average higher $\mathrm{X}$-ray luminosities than steep-spectrum (SS) quasars. The question arises whether the differences in spectral index and luminosity are truly intrinsic or if they can be attributed to a dependence of the source properties on orientation by relativistic beaming. The answer is of importance to test 'unified schemes' (Orr \& Browne 1982) and to understand the X-ray emission of radio quasars. The unified schemes assume that radio sources consist of flat (core/jet) and steep (extended-lobe) radio-emitting components; the differences between FS and SS sources are due to Doppler boosting of the core emission and to projection effects. We composed a sample of 233 radio quasars detected with $R O S A T$, whose radio core fluxes are available from our $V L A$ observations (Laurent-Muehleisen et al. 1996) or from previous VLA and VLBI observations. We used as dividing line between FS and SS sources $\alpha=-0.5\left(S \propto \nu^{\alpha}\right)$.

\section{X-Ray Properties and Radio Morphology}

The core-dominance $R$, the $K$-corrected ratio of core to extended-flux density, is used as indicator of the orientation of the jets in quasars with respect to the line of sight. It is related to the radio spectral index, as FS sources are coredominated (high $R$ ) and SS sources are lobe-dominated (low $R$ ). We confirmed and strengthened the anti-correlation between the soft X-ray power-law photon index $\Gamma$ and $R$, as shown in Fig. 1, which was claimed by Shastri et al. (1993) using Einstein data. The known difference of the mean $\Gamma$ between FS and SS quasars can be attributed to the dependence of $\Gamma$ on $R$, and thus on orientation effects.

The X-ray emission in radio quasars is found to be linked closely to the total radio power. The dependence of $\mathrm{X}$-ray luminosity on radio-core luminosity is, however, different for FS and SS quasars; it is a function of $R$. Applying regression analyses to various $R$ bins yields decreasing slopes $\beta\left(\log L_{x} \propto \beta \log L_{\text {core }}\right.$ ) with the inclusion of lower- $R$ objects. For high- $R$ objects, in which the radio 

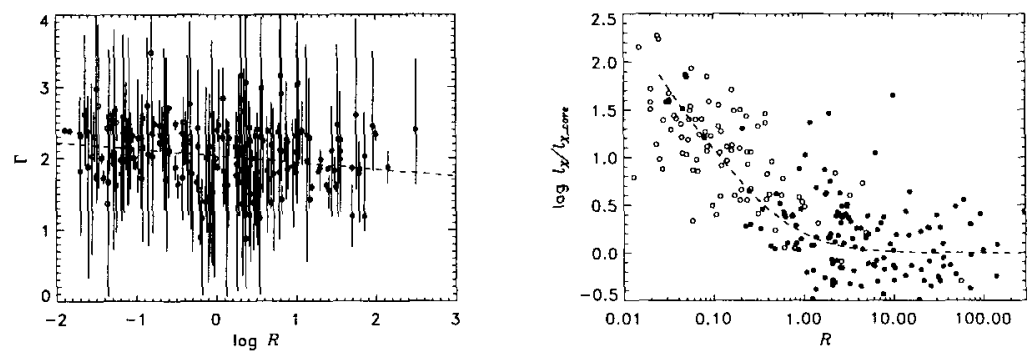

Figure 1. Left: X-ray photon indices vs. $R$; errors are $1 \sigma$. Right: Ratio of the total to estimated core X-ray luminosities vs. $R$.

emission is dominated by the core, the $\mathrm{X}$-ray luminosity is almost linearly correlated $(\beta \approx 0.97 \pm 0.33)$ with the radio-core power. Thus the $\mathrm{X}$-ray emission is closely related to the radio-core emission and is probably beamed as well. The decrease in the slope of $\log L_{x} \propto \log L_{\text {core }}$ relationship towards lower- $R$ objects suggests that the radio core-related component alone cannot account for the total X-ray emission, an alternative component must play a role in objects which are not dominated by the core. To test the dependence of the X-ray luminosity on radio morphology we separated out the X-ray contribution from the radio core-related component using the above $L_{x}($ core $) \propto L_{\text {core }}$ relationship. Figure 2 shows the $\log$ arithmic ratio $\log L_{x} / L_{x}($ core $)$ ) versus $R$, which appears to be strongly anti-correlated, confirming the results of Baker et al. (1995). The dashed line shows the predictions by a two-component model of Kembhavi (1993), which consists of a core and an isotropic component.

\section{Conclusions}

The flattening of the soft X-ray spectral index with increasing core dominance is confirmed. The correlation between the $\mathrm{X}$-ray and radio-core luminosities is dependent on radio morphology; a close proportionality is found for highly core-dominated quasars. Two components of X-ray emission are needed and the composition depends on radio morphology; thus the observed differences in X-rays between FS and SS quasars are attributable to the effect of radio morphology. The data support unification of FS and SS quasars.

\section{References}

Baker, J. C., Hunstead, R. W., \& Brinkmann, W. 1995, MNRAS, 277, 553.

Kembhavi, A. 1993, MNRAS, 264, 683.

Laurent-Muehleisen, S. A., et al. 1996, A\&AS, in press.

Orr, M., \& Browne, I. W. A. 1982, MNRAS, 200, 1067.

Shastri, P., Wilkes, B. J., Elvis, M., \& McDowell, J. 1993, ApJ, 410, 29. 